\title{
RF Photoinjector Based Femtosecond Relativistic Electron Diffraction
}

\author{
P.Musumeci, C. M. Scoby, J. T. Moody, M. Westfall and M. S. Gutierrez \\ Department of Physics and Astronomy, University of California, Los Angeles, CA, 90095-1547
}

Electron diffraction holds the promise to yield real time resolution of atomic motion in an easily accessible environment like a university laboratory at a fraction of the cost than 4th generation $\mathrm{x}$-ray sources. Currently the limit in time-resolution for conventional electron diffraction is set by how short an electron pulse can be made. A possible solution to maintain the highest possible beam intensity without excessive pulse broadening from space charge effects is to increase the electron energy to the $\mathrm{MeV}$ level where relativistic effects significantly reduce the space charge forces allowing for the generation of intense ultrashort beams

Rf photoinjectors can in principle deliver up to $10^{7}-10^{8}$ electrons packed in bunches of $\sim 100 \mathrm{fs}$ length allowing an unprecedented time resolution and enabling the study of irreversible phenomena by single shot diffraction patterns $[1,2]$.

The UCLA Pegasus laboratory has recently demonstrated time resolved single shot Femtosecond Relativistic Electron Diffraction (FRED) using a $\sim 250$ fs long relativistic beam from an $\mathrm{rf}$ photoinjector [3]. The parameters for the Pegasus FRED experiment are reported in Table 1. The significant improvements with respect to previous experiments with relativistic beams which were typically characterized by poor resolution or not sufficient involving a) the use of a collimating hole in front of the sample to remove dark current and high emittance beam halo and $b$ ) an optimization of the sample-screen distance based on the constraints related to the collection efficiency and the detector transverse dimensions.

We use this novel technique to study the evolution of the laser induced solid-liquid transition in metal foils of different thicknesses. An important difference of FRED with respect to conventional UED is the ability to probe deeper in the material. Due to the heat transfer mechanism in metal foils, which at the beginning is dominated by the ballistic electron transport, the energy of the pump laser is distributed over a volume much larger than the one that can be estimated using the optical penetration depth very quickly. Ballistic electrons ranges have been measured to be $>100 \mathrm{~nm}$ in gold for example. The relativistic electrons from the RF photoinjector have a longer penetration depth and can probe the lattice temperature, and the melting phase transition in larger samples. This should shed a light over the kinetics of the melting transition away from ultrathin (20nm and smaller) foils which have been employed so far.

Future directions of FRED include the use of RF deflectors to temporally resolve in a true single shot the structural dynamics of a phase transformation and the development of novel RF structures optimized for the generation of sub-50 fs intense electron beams. 
TABLE 1. Pegasus photoinjector parameters for the single shot high quality diffraction pattern experiment.

\begin{tabular}{ll}
\hline Beam energy & $3.5 \mathrm{MeV}$ \\
Peak field at the cathode & $75 \mathrm{MV} / \mathrm{m}$ \\
Injection phase & $25 \mathrm{deg}$ \\
Bunch length at sample & $250 \mathrm{fs}$ \\
Laser spot size & $500 \mathrm{um}$ \\
Collimating hole diameter & $1 \mathrm{~mm}$ \\
Beam charge & $10 \mathrm{pc}(@$ cathode $) / 1.6 \mathrm{pC}$ (after hole) \\
Cathode sample distance & $0.9 \mathrm{~m}$ \\
Sample-screen distance & $2.4 \mathrm{~m}$ \\
\hline
\end{tabular}

\section{References}

[1] W. E. King et al. J. of Appl. Phys., 97:111101, 2005.

[2] X. J. Wang, Z. Wu, and H. Ihee. In Proceedings of 2003 Particle Accelerator Conference, Portland, Or, 2003.

[3] P. Musumeci et al., Review of Scientific Instruments, 81, 013306 (2010)

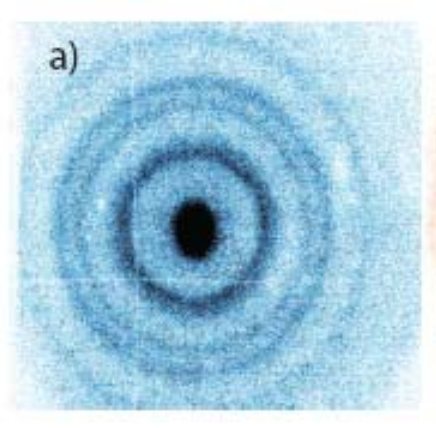

b)

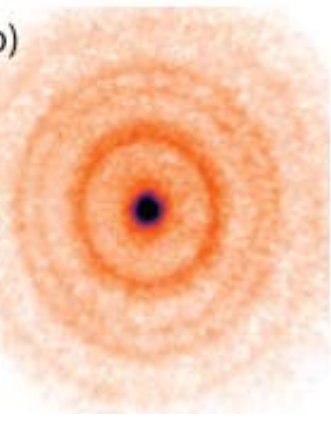

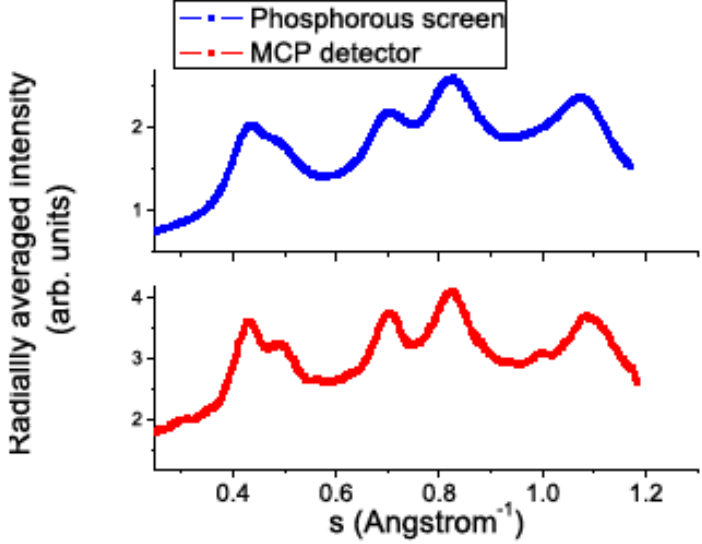

FIG. 1. Single shot diffraction patterns from a $100 \mathrm{~nm}$ thick Au foil obtained a) with the phosphor screen and b) using a microchannel plate detector. 